\title{
NCCN Work Group Report: Emerging Issues in Tissue Allocation
}

Jessica K. DeMartino, $\mathrm{PhD}$

\begin{abstract}
Expanding research interests in molecular profiling over the past several years have led researchers in academia and pharmaceutical and biotechnology companies to significantly increase their need for access to tissue specimens collected through clinical care and clinical trials. As a result, tissue allocation has become a growing issue for many clinical and translational investigators. High-quality biospecimens are needed by all stakeholders in order to have scientifically accurate studies and results. At the center of the process are the patients, who have increasingly become active partners in the clinical research enterprise as individuals and through highly sophisticated patient advocacy organizations. All stakeholders must recognize that human specimens, including tissue, represent a valuable and unique resource that must have proper acquisition, handling, custodianship, and consent for use in accordance with best practices for biospecimen resources.
\end{abstract}

J Natl Compr Canc Netw 2016;14(3):265-271

Biomedical research generates novel information about cancer every day. New scientific capabilities, such as nextgeneration sequencing, provide investigators new ways to study cancer biology, while growing numbers of clinical trials test advanced interventions, such as combination therapies and immunotherapies. These efforts have created increased demand and need for human tissue and blood samples in addition to standard-of-care tissue-based assessments.
Human tissue, however, can be difficult to procure; samples may be limited, scope of use may be limited based on previous consent, competition for available tissue may be fierce, and maintaining tissue for future patient care purposes is not only desirable but also mandated by standards of care in pathology and federal regulations. Although obtaining tissue from patients may have associated risk and discomfort, individual pa-

\section{NCCN Tissue Allocation Work Group}

David L. Rimm, MD, PhD, Co-Chair*

Yale Cancer Center/Smilow Cancer Hospital

Daniel Sullivan, MD, Co-Chair

Moffit Cancer Center

Al B. Benson III, MD

Robert H. Lurie Comprehensive Cancer Center of

Northwestern University

Judith Carrithers, JD, MPA, CIP*

The Sidney Kimmel Comprehensive Cancer Center at

Johns Hopkins

Jeffrey W. Clark, MD

Massachusetts General Hospital Cancer Center

Carolyn Compton, MD, PhD*

National Biomarker Development Alliance

Marisa Dolled-Filhart, PhD*

Merck
Thomas J. Flotte, MD*

Mayo Clinic Cancer Center

Karen Hansen

Fred Hutchinson Cancer Research Center/

Seattle Cancer Care Alliance

Nadia Haque, PhD

Genentech

Laura Lyman Rodriguez, PhD

National Human Genome Research Institute

Wendy Selig*

WSCollaborative

Alexa Sirko-Osadsa, PhD*

Novartis Institutes for BioMedical Research

NCCN Staff: Marian Birkeland, PhD*; Robert Carlson, MD;

Patricia Goelz, MPH*; Joan McClure, MS; Elizabeth Nardi, MS*; and Diane Paul, MS, RN*

*Writing/Editorial Contributor

This activity is supported by ApoBiologix, a Division of Apotex Corp; Astellas; Biodesix, Inc.; Gilead Sciences, Inc.; HELSINN; Incyte Corporation; Janssen; Janssen Diagnostics, LLC; Medivation Inc.; Nanostring; Onyx Pharmaceuticals, Inc., an Amgen subsidiary; Pharmacyclics LLC, an AbbVie Company; Takeda Oncology; and Teva Oncology. Sponsored by Boehringer Ingelheim Pharmaceuticals, Inc. and Novartis Oncology. Supported by a grant from ImmunoGen Inc. This activity is supported by a contribution from Lilly. 
tients and patient advocacy organizations are often eager to participate as true partners in the clinical research enterprise. The prevailing belief among all stakeholders is that, first and foremost, the priority for use of human tissue should be for diagnostic/clinical purposes. It is only after clinical need is met that tissue should be made available for research purposes. Investigators desiring tissue for research may include the patient's treatment institution, other institutions, or companies that develop drugs, devices, or companion diagnostics. Uses of the tissue may range from screening for clinical trial enrollment, exploratory biomarker analyses, and biobanking for future research and biomarker development.

Tissue samples, whether for research or clinical diagnosis, are often housed in a biorepository or clinical archive, where biological materials are collected, processed, stored, and distributed in support of future scientific investigation. The biorepository assures the proper consent, clinical provenance, annotation, and quality of the biospecimens in its collection and manages the accessibility and distribution/ disposition of samples and data. Large cancer centers often have a shared resource that provides banked tissues to investigators while maintaining patient confidentiality. Pharmaceutical companies and other entities may also maintain their own biorepositories. In general, biorepositories are required to be overseen by a committee that governs how the tissue and other samples are allocated to investigators and reviews specific proposals to assure that tissue requests are appropriate for a proposed study and in line with patient consent. However, even though biorepositories can enable greater sharing of resources, there are not enough specimens to meet the demand. As a result, tissue allocation has become a growing issue for many clinical and translational investigators.

Biospecimens are vulnerable to environmental and biological stresses introduced by routine collection, processing, storage, and transport procedures prior to analysis. ${ }^{1}$ This preanalytical variation may transform the molecular composition of the biospecimen before it ever reaches the clinician or researcher. Preanalytic variables include medical or surgical interventions before the specimen is removed from the patient, processing methods, and specimen transport and storage. Without proper understanding of the impact of these sources of variability, molecular changes may be misinterpreted as disease-related or even disease-specific findings. Thus, optimum collection, processing, storage, tracking, and shipment of biospecimens are central to ensuring reproducible and valid outcomes of clinical studies. Clinically, there is a potential for incorrect diagnosis or treatment. In research, there is increased risk of irreproducible results and misinterpretation of artifacts as biomarkers when preanalytic variables are not controlled.

Additionally, tumor biology is complex. Mutations found in single biopsies may not be found in other regions of the same tumor, and "favorable" and "unfavorable" molecular profiles may be generated by analysis of different regions of the same tumor. ${ }^{2}$ Different parts of the same tumor can have different mutations in the very same genes, resulting in local heterogeneity, whereas treatment often creates evolutionary pressures that increase heterogeneity.

A multitude of stakeholders, including academic clinicians and researchers, pharmaceutical industry scientists, individual patients, and patient advocacy organizations, have a vested interest in the sharing of human biospecimens. These stakeholders share goals for these biospecimens, including high-quality samples to serve both diagnostic and research purposes, and sufficient quantity of biospecimen for all planned and consented uses. In order to achieve these goals, new practices must be agreed upon and adopted, including consistent and standard sample collection, processing, storage, and release, and adoption of guidelines on sample control and ownership and also guidelines covering best practices in length of sample retention and uses outside of specified trials.

There are several key challenges to optimizing the use and usefulness of biospecimens. High-quality biospecimens are needed by all stakeholders in order to generate scientifically accurate studies and results, but who will define the quality standards? How can consistent collection of high-quality samples be ensured? Several process issues must be addressed, including improvement of preanalytic processes and sample collection, documentation and sample collection attribution, and management of competing priorities for tissue use.

To identify and examine challenges in tissue collection, processing, storage, and allocation, NCCN convened the NCCN Tissue Allocation work group comprised of clinical research, pathology, and ethics thought leaders from NCCN Member Institutions, including clinicians, pathologists, clinical and 
translational investigators, and Institutional Review Board (IRB) representatives, as well as representatives from industry, professional organizations, and patient advocacy groups. The work group met on March 30, 2015, in Philadelphia. In addition, NCCN held the NCCN Policy Summit: Emerging Issues in Tissue Allocation on June 8, 2015, in Washington, DC. This summit included additional thought leaders representing the aforementioned groups and relevant stakeholders.

This article focuses on 3 topics identified as critical by the work group and discussed at the policy summit: informed consent, preanalytic standards, and tissue sharing between academia and industry.

\section{Informed Consent}

Informed consent is designed to present potential human research participants with sufficient information, including anticipated procedures, risks, and benefits, to make an informed decision about whether to participate in research studies. Obtaining informed consent for the collection, storage, and future research use of biospecimens can be challenging, because the specifics of the future research often are not known at the time of biospecimen collection.

Advances in genome sequencing technology, evolution in our understanding of the genome, the increased power of data storage and sharing, and varying attitudes about genomic privacy necessitate a flexible approach to communicating with prospective participants during the informed consent process and within the consent form. ${ }^{3}$

A perspective published in the New England Journal of Medicine highlights the benefits and drawbacks of various types of consent as applied to biospecimens stored and used in studies unspecified at the time of tissue collection. ${ }^{4}$ Specific consents require that participants are contacted and re-consented for each future study: tiered consents give research participants a variety of choices when samples are collected, and allow participants to express whether they permit their samples to be used in future studies; general permission asks that participants permit all future use of their tissue or sample at the time of initial consent; and with presumed consent, participants in a research study are informed that unless they deny their permission, their samples will be used for additional research.
The NCCN Tissue Allocation Work Group recognized the importance of developing standardized language for informed consent documents (ICDs) related to use of subject tissue. Often, subjects are asked to sign separate ICDs for use of their tissue, which may not be consistent with other ICDs they have signed for trial participation. Integrating standardized language that addresses ownership, how tissue will be used in research and nonresearch settings, how it will be used internally and externally, whether the tissue will be stored in a biorepository for future research, and whether the patient will be informed of future use of the tissue was strongly recommended.

IRBs play an important role in ensuring compliance with regulations, which includes a review of ICDs. IRBs are facing challenges as they are required to review increasingly larger numbers of research studies using subject tissue. Uniformity and standardization in ICD language therefore becomes critical in helping to streamline the review process while ensuring protection of human research subjects.

Tiered consent is an option when obtaining consent from patients. In a tiered consent form, research participants are presented with a menu of options, which may include general permission for future use; consent only for future uses related to the original study topic; consent for future uses unrelated to original study topic; or specification that the investigators must obtain specific consent for any future use that differs from the original study. ${ }^{4}$ Tiered informed consent for the use of tissue in future research is challenging and was discouraged by the NCCN Working Group. These consents are onerous to adjudicate, are difficult to track for compliance, make re-consent of subjects nearly impossible, and may potentially erode public trust in research if the consent process is not conducted properly. As broad consent for unspecified research is considered acceptable by the Office of Human Research Protections, the NCCN Working Group recommended developing comprehensive language in consents that pertains to future research.

An alternate method to obtaining informed consent was also discussed at the summit, as research has shown that many subjects are not aware of what they are agreeing to when giving consent. The concept of subjects giving general permission for future use of tissue was posed, because the exact research uses for excess tissue are often unknown and cannot be clearly defined in an ICD. Instead of giving 
consent for unknown future uses, subjects would give general permission for use of their tissues for research purposes that are deemed scientifically meritorious and ethically defensible, and mechanisms for keeping subjects informed of these uses could be put in place, such as a newsletter. Indeed, subjects could give both consent and permission: specific consent for the known uses of tissue and general permission for the unknown uses.

After the summit, the US Department of Health and Human Services (HHS) and 15 other federal departments and agencies announced proposed revisions to the regulations for protection of human subjects in research (ie, the Common Rule). A Notice of Proposed Rulemaking (NPRM) was published in the Federal Register on September 8, 2015.5 These revisions, if approved, would generally require informed consent for the use of stored biospecimens in secondary research (eg, part of a blood sample that is left over after being drawn for clinical purposes), even if the biospecimen is de-identified or anonymized. Broad consent (ie, consent for future, unspecified research studies) would be obtained for the storage and eventual research use of biospecimens. The NPRM states that the Secretary of HHS will promulgate a broad consent template for this purpose.

Lastly, the process of obtaining informed consent should include education and information about all the issues related to tissue allocation at an educational level understood by subjects. The work group believed that most research subjects want their tissue to be used "wisely and well" to advance science, and are willing to give consent to have it used in future research.

\section{Efforts to Improve Preanalytic Variation in Human Biospecimens}

Much discussion at the NCCN Work Group meeting and policy summit focused on preanalytic standards and their importance to biospecimen science and research performed with biospecimens. Several different groups have made, or are currently undertaking, efforts to address this critical issue.

The NCI, through its Biorepositories and Biospecimen Research Branch (BBRB), is leading a national initiative to systematically address and resolve the limited availability of carefully collected and controlled high-quality human biospecimens annotated with essential clinical data and properly consented for broad investigational use. ${ }^{6}$ This issue has been repeatedly identified by the scientific community as a leading obstacle to progress in postgenomics cancer research.

NCI established the Office of Biorepositories and Biospecimen Research (OBBR) in 2005, and the Biorepository Coordinating Committee (BCC), which has an advisory role to the OBBR. The OBBR's mission is to facilitate cancer and biomedical research by improving the quality and consistency of human biospecimens. The objective of the BCC is to coordinate efforts to improve the availability and quality of human specimens needed for research supported by the NCI.

In early 2006, the Biospecimen Research Network (BRN) was initiated to systematically address the impact of specific variables in individual specimen types on molecular data from given analysis platforms. ${ }^{8}$ The goal of the BRN is to address these issues by sponsoring, conducting, and collaborating on studies to assess the effects of human specimen preanalytical variables on the outcome of genomic and proteomic studies conducted for clinical diagnosis and cancer research purposes. By communicating the results of this research to the scientific community, the BRN aims to significantly improve the quality of NCI-funded biospecimen-based research.

The BBRB's Biospecimen Pre-analytical Variables (BPV) program is designed to systematically investigate the effects of individual preanalytical factors on the molecular profiles of biospecimens. ${ }^{9}$ The results of this study will be used to develop evidencebased protocols and best practices for optimal collection, processing, and storage of biospecimens. Moreover, accumulated data from these activities will be widely disseminated to the research community and strengthen the standards for human biobanking.

Highly annotated biospecimens are collected for the BPV program through a tightly regulated infrastructure. Patients are screened, consented, and enrolled in the BPV study at 4 Biospecimen Source Sites (BSSs): Boston Medical Center, Emory University, University of New Mexico, and University of Pittsburgh Medical Center. Currently, the BPV program is collecting tumor and blood specimens to evaluate the effects of several preanalytical factors on downstream molecular analysis results, including the effects of variations in

- cold ischemia time of tumor tissue

- formalin fixation time 
- method of freezing tumor tissue

- storage temperature of frozen tumor tissue

- frozen storage temperature of plasma

An additional initiative of the BBRB is the Biospecimen Research Database (BRD). ${ }^{10}$ The BRD is a free and publicly accessible database that contains peer-reviewed primary and review articles and standard operating procedures (SOPs) in the field of human biospecimen science. This resource was borne out of work done with the RAND Corporation. ${ }^{11}$ OBBR asked RAND to identify and analyze existing data on the effects of variables on biospecimens used to study genetic and proteomic changes in cancer. To make the findings of this project useful to the scientific community, it was necessary to develop a systematic method to capture the wealth of data collected through the review of the scientific literature. A data-curation tool, the BRD, was developed to provide a standardized way of consistently recording data obtained through the literature review. The BRD has evolved to include SOPs as well.

Although the NCI's efforts to improve the quality of biospecimens are impressive, there has been only modest change at the level of individual or institutional practice. Perhaps the most significant change is the inclusion of a 1 -hour maximum time to fixation stipulated in the ASCO/College of American Pathologists (CAP) guidelines for breast cancer markers. ${ }^{12,13}$ A number of papers have been published that address these issues, but none have changed practice. ${ }^{14-18}$ More work and guidance are needed to make an impact on the individual and hospital practice levels of tissue collection and processing.

In order to address this challenge, in December 2014 the National Biomarker Development Alliance (NBDA) hosted 2 convergence conferences ("Converging on Biospecimen Standards for Genomics" and "Converging on Biospecimen Standards for Proteomics"), both co-chaired by Carolyn Compton, $\mathrm{MD}, \mathrm{PhD}$, former director of OBBR, that brought together stakeholders from all realms of translational research and molecular medicine, ${ }^{19}$ including experts and thought leaders in pathology and laboratory medicine, surgery, clinical trials, genomics, proteomics, medical care delivery and reimbursement, government regulation, biomarker research funding, professional medical societies that set and enforce standards of care, and patient advocacy.
The goals of these conferences were to define and implement the procedures and practices that would assure uniform quality, consistency, and "fitness" for the purpose of cutting edge genomic and proteomic molecular analysis of biospecimens from all patients. It was recognized that the achievement of this goal would not only raise the bar for standard of care in clinical medicine, but also revolutionize all aspects of translational research using human samples. Implementation of these practices would improve the quality of medical care by improving the quality and consistency of human biospecimens of all patient samples.

The conferences produced agreement on the "Top 10" preanalytical variables for human tissue and blood that have the greatest compromising effect on the quality and reproducibility of molecular data. The "Top 10 List" for tissue includes time to stabilization; method of processing; method of stabilization; metadata collected; and storage conditions. Once all preanalytical variables are validated and the corresponding performance metrics to control them adequately are defined, the CAP has agreed to implement and enforce these standards in pathology departments and commercial laboratories throughout the country and internationally through their Laboratory Accreditation Program (LAP). Heretofore, no other group or groups, public or private, have been able to accomplish such a feat. The NBDA and the CAP believe that this sweeping reform will create and assure the requisite level of molecular quality of human biospecimens needed to advance precision medicine in the clinic and the research laboratory simultaneously. Toward this end, the CAP and the NBDA have completed a Memorandum of Understanding to work together to define the requisite educational and practice goals necessary to achieve the objective. The CAP has established a special project task force titled "Specimen Standards for Precision Medicine," chaired by Dr. Compton and including membership from multiple Scientific Resource Committees of CAP, that is undertaking a comprehensive review of published data and clinical experience that support the "Top 10 " list of variables; designing and implementing a comprehensive educational program for the clinical and research communities on the issue; and working with the CAP LAP to develop the performance metrics needed for enforcement of control of the 
key variables in accredited laboratories. The target date for completion of this effort is 2017.

Although the work group and summit attendees were very interested and supportive of NBDA's efforts to address preanalytical variables, some concerns were expressed. Increased demand for tissue has not been supported by the increases in funding or manpower needed to ensure high-quality tissue. Implementing preanalytic standards would require additional financial and manpower resources. There are costs associated with having the right people in place at all times to ensure that standards will be met for each biospecimen. Implementing such requirements in a community setting may be difficult, because community pathologists have many different responsibilities and could not be devoted to preanalytic standards for all biospecimens. Financial resources would need to be increased for both academic and community hospitals to implement such programs and requirements. All this said, the ability to implement precision medicine for patient care is dependent on the ability to achieve such standards. Attendees at the summit questioned where the additional financial resources would come from. Panelists expressed doubt that any payer, public or private, would fund this type of mandate. Although all stakeholders recognized that quality data relies on biospecimens of known, consistent quality, there was no agreement as to how best to pay for such programs and standards.

\section{Academia's and Industry's Tissue Relationship}

Challenges regarding how academic researchers and their industry partners share biospecimens were discussed at both the NCCN Work Group meeting and the NCCN Policy Summit. Both stakeholder groups realize they must find ways to share the limited resource of biospecimens that allows both parties to conduct clinical care, research, and drug development.

A major point of contention is the physical form (eg, blocks vs slides) in which tissue is shared with industry. Industry researchers prefer and often request entire tissue blocks, whereas academic researchers rarely, if ever, relinquish entire blocks of tissue. Academic researchers expressed frustration at the high number of slides requested by industry; honoring requests for a high volume of slides would subsequently deplete the block, leaving little or no tissue for future diagnostic needs.

The value and use of cut slides were also discussed. Although an industry researcher may ask for 20 slides, only 5 are immediately used, calling into question whether the other 15 slides are used before degradation takes place. As a potential solution to this problem, David Rimm, MD, discussed the technique of coring a block and providing a reembedded core to those requesting formalin-fixed, paraffin-embedded tissue. The 3- to 4-mm core provides enough tissue to isolate DNA or RNA and can be either sectioned as is or cored up to 4 times with a 0.6 -mm needle for tissue microarray construction. ${ }^{20}$ This method allows for users to store the biospecimen core without presectioning slides.

Academic researchers often feel that the high demand for slides is motivated by industry researchers' desires to use a few slides for current studies and store remaining slides for future, undetermined uses. Improved communication between academic and industry researchers would allow tissue providers to have a clearer understanding of the use of each slide. Although industry demand for tissue varies by research area and the intent of the research, industry researchers may request more tissue upfront because of the concern that tissue will not be available later to conduct subsequent research. Additionally, because of the heterogeneity of a tumor, industry researchers often ask for more slides than necessary to ensure they receive sufficient usable material.

Ultimately, both stakeholder groups agreed that (1) improved communication around planned and future use of biospecimens is needed, and (2) adherence to preanalytic standards will ensure higher-quality biospecimens and subsequently lessen demand for high volumes of tissue by industry researchers.

\section{Closing Statement}

Progress in cancer research has brought new energy to all stakeholders to accelerate the pace of discovery and translation and deliver improved outcomes for patients. Expanding research interests in molecular profiling have led all researchers to significantly increase their use of biospecimens. The limited availability of biospecimens has forced a conversation among stakeholders about improved processes 
for the sharing of this valuable resource. Although biospecimens are limited, prior to analysis they are also vulnerable to environmental and biological stresses during routine collection, processing, storage, and transport. The NCCN Tissue Allocation Work Group identified 3 key areas to address to improve the use of biospecimens: informed consent, preanalytic standards, and the academia-industry relationship. Both the work group and policy summit highlighted that the most important area to address is the development and implementation of preanalytic standards for tissue procurement and storage. Although doing so will take both financial and moral fortitude, the resulting high-quality biospecimens will continue to increase the understanding of human biology and advance cancer treatment on behalf of today's and tomorrow's patients.

\section{References}

1. Moore HM, Kelly A, Jewell SD, et al. Biospecimen reporting for improved study quality. Biopreserv Biobank 2011;9:57-70.

2. Gerlinger M, Rowan AJ, Horswell S, et al. Intratumor heterogeneity and branched evolution revealed by multiregion sequencing. N Engl J Med 2012;366:883-892.

3. Informed Consent for Genomics Research. Available at: https://www. genome.gov/27026588. Accessed August 21, 2015.

4. Mello MM, Wolf LE. The Havasupai indian tribe case-lessons for research involving stored biologic samples. N Engl J Med 2010;363:204-207.

5. Federal Register, Federal Policy for the Projection of Human Subjects. Available at: http://www.gpo.gov/fdsys/pkg/FR-2015-09-08/pdf/201521756.pdf. Accessed September 17, 2015.

6. Biorepositories and Biospecimens Research Branch, Mission and Goals. Available at: http://biospecimens.cancer.gov/about/overview.asp. Accessed August 20, 2015.
7. Biorepository Coordinating Committee. Available at: http://biospecimens. cancer.gov/about/bcc.asp. Accessed August 20, 2015.

8. Biospecimen Research Network. Available at: http://biospecimens.cancer. gov/researchnetwork/default.asp Accessed August 20, 2015.

9. Biospecimen Pre-analytics Variable Program. Available at: http:// biospecimens.cancer.gov/programs/bpv/default.asp. Accessed August 20, 2015.

10. The Biospecimen Research Database. Available at: https://brd.nci.nih.gov/ brd/. Accessed August 20, 2015.

11. Eiseman E. Effects of preanalytical variables on the quality of biospecimens used to study genetic changes in cancer. RAND Corporation 2009. Available: http://www.rand.org/pubs/documented_briefings/DB572.html. Accessed August 20, 2015.

12. Hammond ME, Hayes DF, Dowsett M, et al. American Society of Clinical Oncology/College of American Pathologists guideline recommendations for immunohistochemical testing of estrogen and progesterone receptors in breast cancer. Arch Pathol Lab Med 2010;134:907-922.

13. Wolff AC, Hammond ME, Hicks DG, et al. Recommendations for human epidermal growth factor receptor 2 testing in breast cancer: American Society of Clinical Oncology/College of American Pathologists clinical practice guideline update. J Clin Oncol 2013;31:3997-4013.

14. Khoury T, Sait S, Hwang H, et al. Delay to formalin fixation effect on breast biomarkers. Mod Pathol 2009;22:1457-1467.

15. Yildiz-Aktas IZ, Dabbs DJ, Bhargava R. The effect of cold ischemic time on the immunohistochemical evaluation of estrogen receptor, progesterone receptor, and HER2 expression in invasive breast carcinoma. Mod Pathol 2012;25:1098-1105.

16. Neumeister VM, Anagnostou V, Siddiqui S, et al. Quantitative assessment of effect of preanalytic cold ischemic time on protein expression in breast cancer tissues. J Natl Cancer Inst 2012;104:1815-1824.

17. Vassilakopoulou M, Parisi F, Siddiqui S, et al. Preanalytical variables and phosphoepitope expression in FFPE tissue: quantitative epitope assessment after variable cold ischemic time. Lab Invest 2015;95:334-341.

18. Neumeister VM, Parisi F, England AM, et al. A tissue quality index: an intrinsic control for measurement of effects of preanalytical variables on FFPE tissue. Lab Invest 2014;94:467-474.

19. The National Biomarker Development Alliance Convergence Conferences. Available at: http://nbdabiomarkers.org/projects-standardsand-guidelines/consensus-conferences. Accessed August 20, 2015.

20. Rimm DL, Nielsen TO, Jewell SD, et al. Cancer and leukemia group $\mathrm{B}$ pathology committee guidelines for tissue microarray construction representing multicenter prospective clinical trial tissues. J Clin Oncol 2011;29:2282-2290. 